\title{
Chaos in Duopoly Games via Furstenberg Family Couple
}

\author{
Yu Zhao and Risong Li $\mathbb{D}$ \\ School of Mathematics and Computer Science, Guangdong Ocean University, Zhanjiang 524025, China \\ Correspondence should be addressed to Risong Li; gdoulrs@163.com
}

Received 4 July 2019; Accepted 25 October 2019; Published 23 November 2019

Academic Editor: Marcin Mrugalski

Copyright ( 2019 Yu Zhao and Risong Li. This is an open access article distributed under the Creative Commons Attribution License, which permits unrestricted use, distribution, and reproduction in any medium, provided the original work is properly cited.

\begin{abstract}
Assume that $H_{1}$ and $H_{2}$ are two given closed subintervals of $\mathbb{R}$ and that $f_{2}: H_{1} \longrightarrow H_{2}$ and $f_{1}: H_{2} \longrightarrow H_{1}$ are continuous maps. Let $\Upsilon\left(h_{1}, h_{2}\right)=\left(f_{1}\left(h_{2}\right), f_{2}\left(h_{1}\right)\right)$ be a Cournot map over the space $H_{1} \times H_{2}$. In this paper, we study $\left(\mathscr{G}_{1}, \mathscr{G}_{2}\right)$-chaos (resp. strong $\left(\mathscr{G}_{1}, \mathscr{G}_{2}\right)$-chaos) of such a Cournot map. We will show that the following are true: (1) $\Upsilon$ is $\left(\mathscr{G}_{1}, \mathscr{G}_{2}\right)$-chaotic (resp. strong $\left(\mathscr{G}_{1}, \mathscr{G}_{2}\right)$-chaotic) if and only if $\left.\Upsilon^{2}\right|_{\Lambda_{1}}$ is $\left(\mathscr{G}_{1}, \mathscr{G}_{2}\right)$-chaotic (resp. strong $\left(\mathscr{G}_{1}, \mathscr{G}_{2}\right)$-chaotic) if and only if $\left.\Gamma^{2}\right|_{\Lambda_{2}}$ is $\left(\mathscr{G}_{1}, \mathscr{G}_{2}\right)$-chaotic (resp. strong $\left(\mathscr{G}_{1}, \mathscr{G}_{2}\right)$-chaotic). (2) $\Upsilon$ is $\left(\mathscr{G}_{1}, \mathscr{G}_{2}\right)$-chaotic (resp. strong $\left(\mathscr{G}_{1}, \mathscr{G}_{2}\right)$-chaotic) if and only if $\left.\Upsilon^{2}\right|_{\Lambda_{1} \cup \Lambda_{2}}$ is $\left(\mathscr{G}_{1}, \mathscr{G}_{2}\right)$-chaotic (resp. strong $\left(\mathscr{G}_{1}, \mathscr{G}_{2}\right)$-chaotic). (3) $f_{1} \circ f_{2}$ is $\left(\mathscr{G}_{1}, \mathscr{G}_{2}\right)$-chaotic (resp. strong $\left(\mathscr{G}_{1}, \mathscr{G}_{2}\right)$-chaotic) if and only if so is $f_{2} \circ f_{1} . M R(2000)$ Subject Classification: Primary 37D45, 54H20, and 37B40 and Secondary 26A18 and $28 \mathrm{D} 20$.
\end{abstract}

\section{Introduction}

Let $H_{1}$ and $H_{2}$ be closed subintervals of $\mathbb{R}$, and let $f_{1}$ : $H_{2} \longrightarrow H_{1}$ and $f_{2}: H_{1} \longrightarrow H_{2}$ be continuous. In the whole paper, $\Upsilon: H_{1} \times H_{2} \longrightarrow H_{1} \times H_{2}$ is defined by $\Upsilon\left(h_{1}, h_{2}\right)=\left(f_{1}\left(h_{2}\right), f_{2}\left(h_{1}\right)\right)$ for any $\left(h_{1}, h_{2}\right) \in H_{1} \times H_{2}$. Such a map has been investigated to give a mathematical analysis of Cournot duopoly (see [1]). Probably the first notion of chaos in a mathematically rigorous way was posed by Li and Yorke [2]. Since then, a lot of different notions of chaos have been posed. Akin and Kolyada gave the concept of Li-Yorke sensitivity for the first time [3]. They also gave the concept of spatiotemporal chaos. Schweizer and Smítal gave the concept of distributional chaos [4]. We know that distributional chaos is equivalent to positive topological entropy and some other chaotic properties for some particular spaces (see $[4,5]$ ), and that this equivalence relationship will become invalid for some higher dimensional spaces [6] and some zero-dimensional spaces [7]. In [8], Wang et al. gave the definition of distributional chaos with respect to a sequence and got that such chaos is equivalent to Li-Yorke chaos for continuous maps over a closed subinterval. Over the past few decades, people have been paying very close attention to the chaotic properties of Cournot maps (see $[1,9-13])$. From $[1,12]$ one can see that there exist
Markov perfect equilibria processes. That is, two fixed players move alternatively and ensure that any of them chooses the best reply to the previous action of another player. Put $\Lambda_{1}=\left\{\left(f_{1}\left(h_{2}\right), h_{2}\right): h_{2} \in H_{2}\right\}, \quad \Lambda_{2}=\left\{\left(h_{1}, f_{2}\right.\right.$ $\left.\left.\left(h_{1}\right)\right): h_{1} \in H_{1}\right\}$, and $\Lambda_{12}=\Lambda_{1} \cup \Lambda_{2}$. Obviously, $\Upsilon\left(Q_{12}\right)$ $\subset \Lambda_{12}$. The set $\Lambda_{12}$ is said to be a MPE set for $\Upsilon$ (see [9]). Moreover, in [9], the authors studied several kinds of chaos for Cournot maps and obtained that for any definition they considered in [9], and it does not satisfy the condition that $\Upsilon$ is chaotic if and only if so is $\left.\Upsilon\right|_{\Lambda_{12}}$. It is well known that some chaotic properties of Cournot maps have been explored (see [1,12-17]). Recently, Lu and Zhu further studied some chaotic properties of Cournot maps and showed that some chaotic properties of $\left.\Upsilon\right|_{\Lambda_{12}},\left.\Upsilon^{2}\right|_{\Lambda_{1}}$ and $\left.\Upsilon^{2}\right|_{\Lambda_{2}}$ are same. In this paper, it is shown that for any Cournot map $\Upsilon\left(h_{1}, h_{2}\right)=$ $\left(f_{1}\left(h_{2}\right), f_{2}\left(h_{1}\right)\right)$ over the product space $H_{1} \times H_{2}$, the following properties are hold:

(1) $\Upsilon$ is $\left(\mathscr{G}_{1}, \mathscr{G}_{2}\right)$-chaotic (resp. strong $\left(\mathscr{G}_{1}, \mathscr{G}_{2}\right)$-chaotic) if and only if $\left.\Upsilon^{2}\right|_{\Lambda_{1}}$ is $\left(\mathscr{G}_{1}, \mathscr{G}_{2}\right)$-chaotic (resp. strong $\left(\mathscr{G}_{1}, \mathscr{G}_{2}\right)$-chaotic) if and only if $\left.\Gamma^{2}\right|_{\Lambda_{2}}$ is $\left(\mathscr{G}_{1}, \mathscr{G}_{2}\right)$-chaotic (resp. strong $\left(\mathscr{G}_{1}, \mathscr{G}_{2}\right)$-chaotic).

(2) $\Upsilon$ is $\left(\mathscr{G}_{1}, \mathscr{G}_{2}\right)$-chaotic (resp. strong $\left(\mathscr{G}_{1}, \mathscr{G}_{2}\right)$-chaotic) if and only if $\left.\Upsilon^{2}\right|_{\Lambda_{1} \cup \Lambda_{2}}$ is $\left(\mathscr{G}_{1}, \mathscr{G}_{2}\right)$-chaotic (resp. strong $\left(\mathscr{G}_{1}, \mathscr{G}_{2}\right)$-chaotic) 
(3) $f_{1} \circ f_{2}$ is $\left(\mathscr{G}_{1}, \mathscr{G}_{2}\right)$-chaotic (resp. strong $\left(\mathscr{G}_{1}\right.$, $\mathscr{G}_{2}$ )-chaotic) if and only if so is $f_{2} \circ f_{1}$

\section{Preliminaries}

Let $(H, \xi)$ be a compact metric space. A dynamic system $(H, f)$ means that $f$ is a continuous self-map over the space $H$.

Let $f: H \longrightarrow H$ be a map on the space $(H, \xi)$. The map $f$ is chaotic in the sense of Li-Yorke if there is an uncountable set $\mathscr{C} \subset H$ satisfying that for any $h_{1}, h_{2} \in \mathscr{C}$ with $h_{1} \neq h_{2}$ :

$$
\begin{gathered}
\liminf _{l \longrightarrow \infty} \xi\left(f^{l}\left(h_{1}\right), f^{l}\left(h_{2}\right)\right)=0, \\
\limsup _{l \longrightarrow \infty} \xi\left(f^{l}\left(h_{1}\right), f^{l}\left(h_{2}\right)\right)>0 .
\end{gathered}
$$

This uncountable set $\mathscr{C}$ is called a scrambled set of $f$.

An important generalization of Li-Yorke chaos is distributional chaos, which is given in 1994 by Puu and Sushko [1].

Let $(H, \xi)$ be a metric space and $f: H \longrightarrow H$ be continuous. For any $h_{1}, h_{2} \in H$, the upper (lower) distribution function $F_{h_{1} h_{2}}^{*}(t, f)\left(F_{h_{1} h_{2}}(t, f)\right)$ deduced by $\left(h_{1}, h_{2}\right)$ and $f$ is defined by

$$
\begin{aligned}
& F_{h_{1} h_{2}}^{*}(t, f)=\limsup _{m \longrightarrow \infty} \frac{1}{m} \sum_{j=1}^{m} \chi_{[0, t)}\left(\xi\left(f^{j}\left(h_{1}\right), f^{j}\left(h_{2}\right)\right)\right), \\
& F_{h_{1} h_{2}}(t, f)=\liminf _{m \longrightarrow \infty} \frac{1}{m} \sum_{j=1}^{m} \chi_{[0, t)}\left(\xi\left(f^{j}\left(h_{1}\right), f^{j}\left(h_{2}\right)\right)\right),
\end{aligned}
$$

where $\chi_{[0, t)}$ is the characteristic function of the set $[0, t)$. The map $f$ is distributional chaotic if there is an uncountable subset $\mathscr{C} \subset H$ satisfying that for any $h_{1} \neq h_{2} \in \mathscr{C}$, $F_{h_{1} h_{2}}^{*}(t, f)=1(\forall t>0)$, and $\exists t>0$ such that $F_{h_{1} h_{2}}(t, f)=0$. This uncountable subset $\mathscr{C}$ is called a distributionally scrambled set of $f$. And this point pair $\left(h_{1}, h_{2}\right)$ which satisfies the above two conditions is called a distributionally scrambled pair of $f$.

In 1997, Furstenberg family is introduced by Akin [18]. Then, Xiong and Tan defined $\left(\mathscr{G}_{1}, \mathscr{G}_{2}\right)$-chaos and described chaos via Furstenberg family couple. Also, they obtained some sufficient conditions of $\left(\mathscr{G}_{1}, \mathscr{G}_{2}\right)$-chaos (see [19]).

Let $\mathbb{N}=\{1,2,3, \ldots\}, \mathbb{N}_{0}=\mathbb{N} \cup\{0\}$, and $\mathscr{P}$ be the collection of all subsets of $\mathbb{N}_{0}$. A collection $\mathscr{G} \subset \mathscr{P}$ is called a Furstenberg family (see [19]) if it satisfies that if $G_{1} \subset G_{2}$ and $G_{1} \in \mathscr{G}$ then $G_{2} \in \mathscr{G}$. A family $\mathscr{G}$ is said to be proper if it is a proper subset of $\mathscr{P}$ (see [19]). In the whole paper, we suppose that all Furstenberg families are proper. Clearly, a family $\mathscr{G}$ is proper if and only if $\mathbb{N}_{0} \in \mathscr{G}$ and $\varnothing \notin \mathscr{G}$ (see [19]).

For any Furstenberg families $\mathscr{G}_{1}$ and $\mathscr{G}_{2}$ and any map $f: H \longrightarrow H, \mathscr{C} \subset H$ is called a $\left(\mathscr{G}_{1}, \mathscr{G}_{2}\right)$-scrambled set of $f$ (see [19]), if $\forall h_{1} \neq h_{2} \in \mathscr{C}$, the following two conditions are satisfied:

(1) $\forall t>0,\left\{m \in \mathbb{N}: \xi\left(f^{m}\left(h_{1}\right), f^{m}\left(h_{2}\right)\right)<t\right\} \in \mathscr{G}_{1}$

(2) $\exists \delta>0,\left\{m \in \mathbb{N}: \xi\left(f^{m}\left(h_{1}\right), f^{m}\left(h_{2}\right)\right)>\delta\right\} \in \mathscr{G}_{2}$
This pair $\left(h_{1}, h_{2}\right)$ is called a $\left(\mathscr{G}_{1}, \mathscr{G}_{2}\right)$-scrambled pair of $f$. The map $f$ is said to be $\left(\mathscr{G}_{1}, \mathscr{G}_{2}\right)$-chaotic if there is an uncountable $\left(\mathscr{G}_{1}, \mathscr{G}_{2}\right)$-scrambled set of $f$. When $\mathscr{G}_{1}=\mathscr{G}_{2}$ $=\mathscr{G}$, the map $f$ is said to be $\mathscr{G}$-chaotic and the pair $\left(h_{1}, h_{2}\right)$ is a $\mathscr{G}$-scrambled pair. The map $f$ is said to be strong $\left(\mathscr{G}_{1}, \mathscr{G}_{2}\right)$-chaotic if one can find $\delta>0$ satisfying that for any $h_{1} \neq h_{2} \in \mathscr{C}, \forall t>0, \quad\left\{m \in \mathbb{N}: \xi\left(f^{m}\left(h_{1}\right), f^{m}\left(h_{2}\right)\right)<t\right\} \in \mathscr{G}_{1}$ and $\left\{m \in \mathbb{N}: \xi\left(f^{m}\left(h_{1}\right), f^{m}\left(h_{2}\right)\right)>\delta\right\} \in \mathscr{G}_{2}$.

Similarly, one can give the concept of strong $\mathscr{G}$-chaos.

Let $G \subset \mathbb{N}$. The upper density $\bar{\mu}(G)$ and the lower density $\underline{\mu}(G)$ of $G$ are defined by

$$
\begin{aligned}
& \bar{\mu}(G)=\limsup _{m \longrightarrow \infty} \frac{\#(G \cap\{1,2, \ldots, m\})}{m}, \\
& \underline{\mu}(G)=\liminf _{m \longrightarrow \infty} \frac{\#(G \cap\{1,2, \ldots, m\})}{m},
\end{aligned}
$$

where \# $(G)$ denotes the cardinality of the set $G$.

Let $\mathscr{B}$ denotes the set of all infinite subsets of $\mathbb{N}$. For arbitrary $t \in[0,1]$, put $\bar{M}(t)=\{F \in \mathscr{B}: \bar{\mu}(F) \geq t\}$. Then, a pair $\left(h_{1}, h_{2}\right)$ is a $(\bar{M}(0), \bar{M}(0))$-scrambled pair if and only if it is a Li-Yorke scrambled pair (see [19]). A pair $\left(h_{1}, h_{2}\right)$ is a $(\bar{M}(1), \bar{M}(1))$-scrambled pair if and only if it is a distributionally scrambled pair (see [19]). Hence, $(\bar{M}(0)$, $\bar{M}(0))$-chaos is Li-Yorke chaos, and ( $\bar{M}(1), \bar{M}(1))$-chaos is distributional chaos.

For $i \in \mathbb{N}_{0}$ and $G \in \mathscr{P}$, let $G+i=\{j+i: j \in G\} \cap \mathbb{N}_{0}$ and $G-i=\{j-i: j \in G\} \cap \mathbb{N}_{0}$. A Furstenberg family $\mathscr{G}$ is said to be translation-invariant if for any $G \in \mathscr{G}$ and any $i \in \mathbb{N}_{0} G+$ $i \in \mathscr{G}$ and $G-i \in \mathscr{G}$. It is easily seen that $\mathscr{B}$ is a proper and translation-invariant family (see [19]).

Clearly, for any $t \in[0,1], \bar{M}(t)$ is a translation-invariant Furstenberg family and $\bar{M}(0)=\mathscr{B}$ (see [19]).

\section{Main Results}

Theorem 1. Let the product metric $\xi$ on the product space $H_{1} \times H_{2}$ be defined by $\xi\left(\left(a_{1}, b_{1}\right),\left(a_{2}, b_{2}\right)\right)=\max$ $\left\{\left|a_{2}-a_{1}\right|,\left|b_{2}-b_{1}\right|\right\}$ and the product map $\pi_{1} \times \pi_{2}$ of $\pi_{1}: H_{1} \longrightarrow H_{1}$ and $\pi_{2}: H_{2} \longrightarrow H_{2}$ be defined by $\left(\pi_{1} \times\right.$ $\left.\pi_{2}\right)(a, b)=\left(\pi_{1}(a), \pi_{2}(b)\right)$ for any $a \in H_{1}$ and any $b \in H_{2}$, where $H_{1}, H_{2} \subset \mathbb{R}$ are compact intervals, and let $\Upsilon(a, b)=$ $\left(f_{1}(b), f_{2}(a)\right)$ be a Cournot map. If $\mathscr{G}_{1}$ and $\mathscr{G}_{2}$ are two Furstenberg families such that $\mathscr{G}_{2}$ is translation-invariant, then $f_{1} \circ f_{2}$ is $\left(\mathscr{G}_{1}, \mathscr{G}_{2}\right)$-chaotic if and only if so is $f_{2} \circ f_{1}$.

Proof. Suppose that $f_{1} \circ f_{2}$ is $\left(\mathscr{G}_{1}, \mathscr{G}_{2}\right)$-chaotic. By the definition, there is an uncountable $\left(\mathscr{G}_{1}, \mathscr{G}_{2}\right)$-scrambled set $D \subset H_{1}$ of $f_{1} \circ f_{2}$. By the definition, for any given $b>0$ and any $h_{1}, h_{2} \in H_{1}$ with $h_{1} \neq h_{2}$ one has that

$$
\left\{m \in \mathbb{N}:\left|\left(f_{1} \circ f_{2}\right)^{m}\left(h_{1}\right)-\left(f_{1} \circ f_{2}\right)^{m}\left(h_{2}\right)\right|<b\right\} \in \mathscr{G}_{1} \text {. }
$$

As $f_{2}$ is uniformly continuous, for any $a>0$ there is $b>0$ such that $\left|p_{1}-p_{2}\right|<b$ and $p_{1}, p_{2} \in H_{1}$ imply that $\left|f_{2}\left(p_{1}\right)-f_{2}\left(p_{2}\right)\right|<a$. So, if

$$
\left|\left(f_{1} \circ f_{2}\right)^{m}\left(h_{1}\right)-\left(f_{1} \circ f_{2}\right)^{m}\left(h_{2}\right)\right|<b,
$$


then

$$
\left|\left(f_{2} \circ f_{1}\right)^{m}\left(f_{2}\left(h_{1}\right)\right)-\left(f_{2} \circ f_{1}\right)^{m}\left(f_{2}\left(h_{2}\right)\right)\right|<a .
$$

Consequently, by

$$
\begin{array}{r}
\left\{m \in \mathbb{N}:\left|\left(f_{1} \circ f_{2}\right)^{m}\left(h_{1}\right)-\left(f_{1} \circ f_{2}\right)^{m}\left(h_{2}\right)\right|<b\right\} \in \mathscr{G}_{1}, \\
\left\{m \in \mathbb{N}:\left|\left(f_{2} \circ f_{1}\right)^{m}\left(f_{2}\left(h_{1}\right)\right)-\left(f_{2} \circ f_{1}\right)^{m}\left(f_{2}\left(h_{2}\right)\right)\right|<a\right\} \in \mathscr{G}_{1} .
\end{array}
$$

By the definition, for any $h_{1}, h_{2} \in H_{1}$ with $h_{1} \neq h_{2}$ there is $\delta>0$ such that

$$
\left\{m \in \mathbb{N}:\left|\left(f_{1} \circ f_{2}\right)^{m}\left(h_{1}\right)-\left(f_{1} \circ f_{2}\right)^{m}\left(h_{2}\right)\right|>\delta\right\} \in \mathscr{G}_{2} .
$$

As $f_{1}$ is uniformly continuous, for the above $\delta>0$ there is $\delta^{\prime}>0$ such that $\left|p_{1}-p_{2}\right| \leq \delta^{\prime}$ and $p_{1}, p_{2} \in H_{2}$ imply that $\left|f_{1}\left(p_{1}\right)-f_{1}\left(p_{2}\right)\right| \leq \delta$. So, if

$$
\left|\left(f_{1} \circ f_{2}\right)^{m}\left(h_{1}\right)-\left(f_{1} \circ f_{2}\right)^{m}\left(h_{2}\right)\right|>\delta \text {, }
$$

then

$$
\left|\left(f_{2} \circ f_{1}\right)^{m-1}\left(f_{2}\left(h_{1}\right)\right)-\left(f_{2} \circ f_{1}\right)^{m-1}\left(f_{2}\left(h_{2}\right)\right)\right|>\delta^{\prime} .
$$

As $\mathscr{G}_{2}$ is translation-invariant, by

$$
\begin{array}{r}
\left\{m \in \mathbb{N}:\left|\left(f_{1} \circ f_{2}\right)^{m}\left(h_{1}\right)-\left(f_{1} \circ f_{2}\right)^{m}\left(h_{2}\right)\right|>\delta\right\} \in \mathscr{S}_{2}, \\
\left\{m-1 \in \mathbb{N}:\left|\left(f_{2} \circ f_{1}\right)^{m-1}\left(f_{2}\left(h_{1}\right)\right)-\left(f_{2} \circ f_{1}\right)^{m-1}\left(f_{2}\left(h_{2}\right)\right)\right|>\delta^{\prime}\right\}
\end{array}
$$

This means that

$$
\left\{m \in \mathbb{N}:\left|\left(f_{2} \circ f_{1}\right)^{m}\left(f_{2}\left(h_{1}\right)\right)-\left(f_{2} \circ f_{1}\right)^{m}\left(f_{2}\left(h_{2}\right)\right)\right|>\delta^{\prime}\right\} \in \mathscr{G}_{2} .
$$

Thus, Theorem 1 is true.

Theorem 2. Let the product metric $\xi$ on the product space $H_{1} \times H_{2}$ be defined by $\xi\left(\left(a_{1}, b_{1}\right),\left(a_{2}, b_{2}\right)\right)=\max \left\{\mid a_{2}\right.$ $\left.-a_{1}|,| b_{2}-b_{1} \mid\right\}$ and the product map $\pi_{1} \times \pi_{2}$ of $\pi_{1}: H_{1} \longrightarrow H_{1}$ and $\pi_{2}: H_{2} \longrightarrow H_{2}$ be defined by $\left(\pi_{1} \times\right.$ $\left.\pi_{2}\right)(a, b)=\left(\pi_{1}(a), \pi_{2}(b)\right)$ for any $a \in H_{1}$ and any $b \in H_{2}$, where $H_{1}, H_{2} \subset \mathbb{R}$ are compact intervals, and let $\Upsilon(a, b)=$ $\left(f_{1}(b), f_{2}(a)\right)$ be a Cournot map. If $\mathscr{G}_{1}$ and $\mathscr{G}_{2}$ are two Furstenberg families such that $\mathscr{G}_{2}$ is translation-invariant, then $f_{1} \circ f_{2}$ is strong $\left(\mathscr{G}_{1}, \mathscr{G}_{2}\right)$-chaotic if and only if so is $f_{2} \circ f_{1}$.

Proof. Suppose that $f_{1} \circ f_{2}$ is strong $\left(\mathscr{G}_{1}, \mathscr{G}_{2}\right)$-chaotic. By the definition, there is an uncountable strong $\left(\mathscr{G}_{1}\right.$, $\mathscr{G}_{2}$ )-scrambled set $D \subset H_{1}$ of $f_{1} \circ f_{2}$. By the definition, for any given $b>0$ and any $h_{1}, h_{2} \in H_{1}$ with $h_{1} \neq h_{2}$ one has that

$$
\left\{m \in \mathbb{N}:\left|\left(f_{1} \circ f_{2}\right)^{m}\left(h_{1}\right)-\left(f_{1} \circ f_{2}\right)^{m}\left(h_{2}\right)\right|<b\right\} \in \mathscr{G}_{1} \text {. }
$$

As $f_{2}$ is uniformly continuous, for any $a>0$ there is $b>0$ such that $\left|p_{1}-p_{2}\right|<b$ and $p_{1}, p_{2} \in H_{1}$ imply that $\left|f_{2}\left(p_{1}\right)-f_{2}\left(p_{2}\right)\right|<a$. So, if

$$
\left|\left(f_{1} \circ f_{2}\right)^{m}\left(h_{1}\right)-\left(f_{1} \circ f_{2}\right)^{m}\left(h_{2}\right)\right|<b,
$$

then

$$
\left|\left(f_{2} \circ f_{1}\right)^{m}\left(f_{2}\left(h_{1}\right)\right)-\left(f_{2} \circ f_{1}\right)^{m}\left(f_{2}\left(h_{2}\right)\right)\right|<a .
$$

Consequently, by

$$
\left\{m \in \mathbb{N}:\left|\left(f_{1} \circ f_{2}\right)^{m}\left(h_{1}\right)-\left(f_{1} \circ f_{2}\right)^{m}\left(h_{2}\right)\right|<b\right\} \in \mathscr{G}_{1},
$$$$
\left\{m \in \mathbb{N}:\left|\left(f_{2} \circ f_{1}\right)^{m}\left(f_{2}\left(h_{1}\right)\right)-\left(f_{2} \circ f_{1}\right)^{m}\left(f_{2}\left(h_{2}\right)\right)\right|<a\right\} \in \mathscr{G}_{1} \text {. }
$$

By the definition, for any $h_{1}, h_{2} \in H_{1}$ with $h_{1} \neq h_{2}$ there is $\delta>0$ satisfying that for any $h_{1}, h_{2} \in H_{1}$ with $h_{1} \neq h_{2}$, one has that

$$
\left\{m \in \mathbb{N}:\left|\left(f_{1} \circ f_{2}\right)^{m}\left(h_{1}\right)-\left(f_{1} \circ f_{2}\right)^{m}\left(h_{2}\right)\right|>\delta\right\} \in \mathscr{G}_{2} .
$$

As $f_{1}$ is uniformly continuous, for the above $\delta>0$ there is $\delta^{\prime}>0$ such that $\left|p_{1}-p_{2}\right| \leq \delta^{\prime}$ and $p_{1}, p_{2} \in H_{2}$ imply that $\left|f_{1}\left(p_{1}\right)-f_{1}\left(p_{2}\right)\right| \leq \delta$. So, if

$$
\left|\left(f_{1} \circ f_{2}\right)^{m}\left(h_{1}\right)-\left(f_{1} \circ f_{2}\right)^{m}\left(h_{2}\right)\right|>\delta,
$$

then

$$
\left|\left(f_{2} \circ f_{1}\right)^{m-1}\left(f_{2}\left(h_{1}\right)\right)-\left(f_{2} \circ f_{1}\right)^{m-1}\left(f_{2}\left(h_{2}\right)\right)\right|>\delta^{\prime} .
$$

As $\mathscr{G}_{2}$ is translation-invariant, by

$$
\begin{array}{r}
\left\{m \in \mathbb{N}:\left|\left(f_{1} \circ f_{2}\right)^{m}\left(h_{1}\right)-\left(f_{1} \circ f_{2}\right)^{m}\left(h_{2}\right)\right|>\delta\right\} \in \mathscr{G}_{2}, \\
\left\{m-1 \in \mathbb{N}:\left|\left(f_{2} \circ f_{1}\right)^{m-1}\left(f_{2}\left(h_{1}\right)\right)-\left(f_{2} \circ f_{1}\right)^{m-1}\left(f_{2}\left(h_{2}\right)\right)\right|>\delta^{\prime}\right\} \in \mathscr{G}_{2} .
\end{array}
$$

This means that

$$
\left\{m \in \mathbb{N}:\left|\left(f_{2} \circ f_{1}\right)^{m}\left(f_{2}\left(h_{1}\right)\right)-\left(f_{2} \circ f_{1}\right)^{m}\left(f_{2}\left(h_{2}\right)\right)\right|>\delta^{\prime}\right\} \in \mathscr{G}_{2} .
$$

Thus, Theorem 2 is true.

Corollary 1. Let $\Upsilon(a, b)=\left(f_{1}(b), f_{2}(a)\right)$ be a Cournot map on the product space $H_{1} \times H_{2}$. Then, for any $a, b \in[0,1]$, $f_{1} \circ f_{2}$ is $(\bar{M}(a), \bar{M}(b))$-chaotic (resp. strong $(\bar{M}(a)$, $\bar{M}(b))$-chaotic) if and only if so is $f_{2} \circ f_{1}$.

Proof. As $\bar{M}(t)$ is a translation-invariant Furstenberg family for any $t \in[0,1]$, by Theorems 1 and 2 one can see that Corollary 1 holds.

Theorem 3. Let $\Upsilon(a, b)=\left(f_{1}(b), f_{2}(a)\right)$ be a Cournot map on the product space $H_{1} \times H_{2}$. If $\mathscr{G}_{1}$ and $\mathscr{G}_{2}$ are two Furstenberg families such that $\mathscr{G}_{2}$ is translation-invariant and satisfy that for any $k \in\{1,2\}$ and any $G \in \mathscr{G}_{k}$, there is $j \in\{0,1\}$ satisfying that $G_{2, j}:=\{i \in\{0,1, \ldots\}: 2 j+i \in G\}$ $\in \mathscr{G}_{k}$, and that for any $k \in\{1,2\}$ and any $G \in \mathscr{G}_{k}$,

$$
G_{2}:=\{2 i+j \in\{0,1, \ldots\}: j \in\{0,1\}, i \in G\} \in \mathscr{G}_{k},
$$

then $\Upsilon$ is $\left(\mathscr{G}_{1}, \mathscr{G}_{2}\right)$-chaotic if and only if so is $\left.\Upsilon^{2}\right|_{\Lambda_{2}}$. 
Proof. We assume that $\Upsilon$ is $\left(\mathscr{G}_{1}, \mathscr{G}_{2}\right)$-chaotic.

Claim 1. $\Upsilon^{2}$ is $\left(\mathscr{G}_{1}, \mathscr{G}_{2}\right)$-chaotic.

The Proof of Claim 1. Assume that $D \subset H_{1} \times H_{2}$ is a $\left(\mathscr{G}_{1}, \mathscr{G}_{2}\right)$-scrambled set of the system $\left(H_{1} \times H_{2}, \Upsilon\right)$. As $\Upsilon$ and $\Upsilon^{2}$ are uniformly continuous, for any $t>0$ there is $t^{\prime}>0$ satisfying that $h_{1}, h_{2} \in H_{1} \times H_{2}$ and $\xi\left(h_{1}, h_{2}\right)<t^{\prime}$ imply $\xi\left(\Upsilon\left(h_{1}\right), \Upsilon\left(h_{2}\right)\right)<t$ and $\xi\left(\Upsilon^{2}\left(h_{1}\right), \Upsilon^{2}\left(h_{2}\right)\right)<t$. By the hypothesis and the definition, for any $d_{1}, d_{2} \in D$ with $d_{1} \neq d_{2}$, one has that

$$
G=\left\{m \in\{0,1, \ldots\}: \xi\left(\Upsilon^{m}\left(d_{1}\right), \Upsilon^{m}\left(d_{2}\right)\right)<t^{\prime}\right\} \in \mathscr{G}_{1} .
$$

As $\mathscr{G}_{1}$ satisfies that for any $G \in \mathscr{G}_{1}$, there is $j \in\{0,1\}$ satisfying that $G_{2, j}:=\{i \in\{0,1, \ldots\}: 2 j+i \in G\} \in \mathscr{G}_{1}$, by the definition there is $j \in\{0,1\}$ satisfying that

$$
G_{2, j}:=\{i \in\{0,1, \ldots\}: 2 j+i \in G\} \in \mathscr{G}_{1} .
$$

By the above argument, one has that

$G_{2, j} \subset\left\{m \in\{0,1, \ldots\}: \xi\left(\Upsilon^{2 m+j+2-j}\left(d_{1}\right), \Upsilon^{2 m+j+2-j}\left(d_{2}\right)\right)<t\right\}$.

That is,

$$
G_{2, j} \subset\left\{m \in\{0,1, \ldots\}: \xi\left(\Upsilon^{2 m+2}\left(d_{1}\right), \Upsilon^{2 m+2}\left(d_{2}\right)\right)<t\right\} .
$$

So,

$$
\left\{m \in\{0,1, \ldots\}: \xi\left(\Upsilon^{2 m+2}\left(d_{1}\right), \Upsilon^{2 m+2}\left(d_{2}\right)\right)<t\right\} \in \mathscr{G}_{1} .
$$

As $\mathscr{G}_{1}$ is translation-invariant,

$$
\left\{m \in\{0,1, \ldots\}: \xi\left(\Upsilon^{2 m}\left(d_{1}\right), \Upsilon^{2 m}\left(d_{2}\right)\right)<t\right\} \in \mathscr{G}_{1}
$$

By the hypothesis and the definition, for any given $d_{1}, d_{2} \in D$ with $d_{1} \neq d_{2}$ there is $\delta>0$ satisfying that

$$
G^{\prime}=\left\{m \in\{0,1, \ldots\}: \xi\left(\Upsilon^{m}\left(d_{1}\right), \Upsilon^{m}\left(d_{2}\right)\right)>\delta\right\} \in \mathscr{G}_{2} .
$$

As $\mathscr{G}_{2}$ satisfies that for any $G \in \mathscr{G}_{2}$, there is $j \in\{0,1\}$ satisfying that $G_{2, j}:=\{i \in\{0,1, \ldots\}: 2 j+i \in G\} \in \mathscr{G}_{2}$, by the definition there is $j \in\{0,1\}$ satisfying that

$$
G_{2, j}^{\prime}:=\left\{i \in\{0,1, \ldots\}: 2 j+i \in G^{\prime}\right\} \in \mathscr{G}_{2} .
$$

As $\Upsilon$ and $\Upsilon^{2}$ are uniformly continuous, for the above $\delta>0$, there is $\delta^{\prime}>0$ satisfying that $h_{1}, h_{2} \in H_{1} \times H_{2}$ and $\xi\left(h_{1}, h_{2}\right) \leq \delta^{\prime}$ imply $\xi\left(\Upsilon\left(h_{1}\right), \Upsilon\left(h_{2}\right)\right) \leq \delta$ and $\xi\left(\Upsilon^{2}\left(h_{1}\right)\right.$, $\left.\Upsilon^{2}\left(h_{2}\right)\right) \leq \delta$. Clearly,

$$
G_{2, j}^{\prime} \subset\left\{m \in\{0,1, \ldots\}: \xi\left(\Upsilon^{2 m}\left(d_{1}\right), \Upsilon^{2 m}\left(d_{2}\right)\right)>\delta^{\prime}\right\},
$$

which means that $\left\{m \in\{0,1, \ldots\}: \xi\left(\Upsilon^{2 m}\left(d_{1}\right), \Upsilon^{2 m}\left(d_{2}\right)\right)>\delta^{\prime}\right\} \in \mathscr{G}_{2}$.

Thus, Claim 1 holds.

As $\Upsilon^{2}=\left(f_{1} \circ f_{2}\right) \times\left(f_{2} \circ f_{1}\right)$, by hypothesis, Claim 1 , the definition of $\left(\mathscr{G}_{1}, \mathscr{G}_{2}\right)$-chaos, and Theorem 1 and its proof, one can easily verify that $f_{1} \circ f_{2}$ and $f_{2} \circ f_{1}$ are $\left(\mathscr{G}_{1}, \mathscr{G}_{2}\right)$-chaotic.

Assume that $f_{1} \circ f_{2}$ is $\left(\mathscr{G}_{1}, \mathscr{G}_{2}\right)$-chaotic. By the definition, there is an uncountable subset $C \subset H_{1}$ which is $\left(\mathscr{G}_{1}, \mathscr{G}_{2}\right)$-scrambled set of $f_{1} \circ f_{2}$. By the proof of Theorem 1 , $f_{2}(C)$ is an uncountable $\left(\mathscr{G}_{1}, \mathscr{G}_{2}\right)$-scrambled set of $f_{2} \circ f_{1}$. Set $A=\left\{\left(a, g_{2}(a)\right): a \in C\right\}$. Then, $A$ is uncountable. By the above argument, the definition of $\left(\mathscr{G}_{1}, \mathscr{G}_{2}\right)$-chaos and the proof of Theorem 1, it is easy to prove that $A$ is a $\left(\mathscr{G}_{1}, \mathscr{G}_{2}\right)$-scrambled set of $\left.\Upsilon^{2}\right|_{\Lambda_{2}}$.

Now, we assume that $\left.\Upsilon^{2}\right|_{\Lambda_{2}}$ is $\left(\mathscr{G}_{1}, \mathscr{G}_{2}\right)$-chaotic.

Claim 2. $\Upsilon$ is $\left(\mathscr{G}_{1}, \mathscr{G}_{2}\right)$-chaotic.

The Proof of Claim 2. By the hypothesis and the definitions, $\Upsilon^{2}$ is $\left(\mathscr{G}_{1}, \mathscr{G}_{2}\right)$-chaotic. Assume that $D$ is a $\left(\mathscr{G}_{1}\right.$, $\left.\mathscr{G}_{2}\right)$-scrambled set of the system $\left(H_{1} \times H_{2}, \Upsilon^{2}\right)$. As $\Upsilon^{n}$ is uniformly continuous for any $n \in\{0,1\}$, for any $t>0$ there is $t^{\prime}>0$ satisfying that $h_{1}, h_{2} \in H_{1} \times H_{2}$ and $\xi\left(h_{1}, h_{2}\right)<t^{\prime}$ imply $\xi\left(\Upsilon^{n}\left(h_{1}\right), \Upsilon^{n}\left(h_{2}\right)\right)<t$ for any $n \in\{0,1\}$. By hypothesis and the definition, for any $d_{1}, d_{2} \in D$ with $d_{1} \neq d_{2}$, one has that

$$
G=\left\{m \in\{0,1, \ldots\}: \xi\left(\Upsilon^{2 m}\left(d_{1}\right), \Upsilon^{2 m}\left(d_{2}\right)\right)<t^{\prime}\right\} \in \mathscr{G}_{1} .
$$

So, for any $m \in G$ and any $n \in\{0,1\}$ we have that

$$
\xi\left(\Upsilon^{2 m+n}\left(d_{1}\right), \Upsilon^{2 m+n}\left(d_{2}\right)\right)<t .
$$

As $\mathscr{G}_{1}$ satisfies that for any $G \in \mathscr{G}_{1}$,

$$
G_{2}:=\{2 i+j \in\{0,1, \ldots\}: j \in\{0,1\}, i \in G\} \in \mathscr{G}_{1} \text {, }
$$

by the definition we have

$$
G_{2}:=\{2 m+n: m \in G, n \in\{0,1\}\} \in \mathscr{G}_{1} .
$$

Clearly,

$$
G_{2} \subset\left\{m \in\{0,1, \ldots\}: \xi\left(\Upsilon^{m}\left(d_{1}\right), \Upsilon^{m}\left(d_{2}\right)\right)<t\right\} .
$$

This means that

$$
\left\{m \in\{0,1, \ldots\}: \xi\left(\Upsilon^{m}\left(d_{1}\right), \Upsilon^{m}\left(d_{2}\right)\right)<t\right\} \in \mathscr{G}_{1} \text {. }
$$

By the hypothesis and the definition, for any given $d_{1}, d_{2} \in D$ with $d_{1} \neq d_{2}$ there is $\delta^{\prime}>0$ satisfying that

$$
G^{\prime}=\left\{m \in\{0,1, \ldots\}: \xi\left(\Upsilon^{m}\left(d_{1}\right), \Upsilon^{m}\left(d_{2}\right)\right)>\delta^{\prime}\right\} \in \mathscr{G}_{2} .
$$

As $\Upsilon^{n}$ is uniformly continuous for any $n \in\{0,1\}$, for the above $\delta^{\prime}>0$ there is $\delta>0$ satisfying that $h_{1}, h_{2} \in H_{1} \times H_{2}$ and $\xi\left(\Upsilon^{2}\left(h_{1}\right), \Upsilon^{2}\left(h_{2}\right)\right)>\delta$ imply $\xi\left(\Upsilon^{n}\left(h_{1}\right), \Upsilon^{n}\left(h_{2}\right)\right)>\delta$ for any $n \in\{0,1\}$. So, for any $m \in G^{\prime}$ and any $n \in\{0,1\}$ we have that 


$$
\xi\left(\Upsilon^{2(m-1)+n}\left(d_{1}\right), \Upsilon^{2(m-1)+n}\left(d_{2}\right)\right)>\delta .
$$

As $\mathscr{G}_{2}$ is translation-invariant, $G^{\prime}-1 \in \mathscr{G}_{2}$. As $\mathscr{G}_{2}$ satisfies that for any $G \in \mathscr{G}_{2}$,

$$
\begin{aligned}
G_{2} & :=\{2 i+j \in\{0,1, \ldots\}: j \in\{0,1\}, i \in G\} \in \mathscr{G}_{2}, \\
\left(G^{\prime}-1\right)_{2} & :=\left\{2(m-1)+n: m-1 \in G^{\prime}-1, n \in\{0,1\}\right\} \in \mathscr{G}_{2} .
\end{aligned}
$$

Clearly,

$$
\left(G^{\prime}-1\right)_{2} \subset\left\{m \in\{0,1, \ldots\}: \xi\left(\Upsilon^{m}\left(d_{1}\right), \Upsilon^{m}\left(d_{2}\right)\right)>\delta\right\},
$$

which means that

$$
\left\{m \in\{0,1, \ldots\}: \xi\left(\Upsilon^{m}\left(d_{1}\right), \Upsilon^{m}\left(d_{2}\right)\right)>\delta\right\} \in \mathscr{G}_{2} .
$$

Thus, Claim 2 holds.

Consequently, Theorem 3 is true.

Theorem 4. Let $\Upsilon(a, b)=\left(f_{1}(b), f_{2}(a)\right)$ be a Cournot map on the product space $H_{1} \times H_{2}$. If $\mathscr{G}_{1}$ and $\mathscr{G}_{2}$ are two Furstenberg families such that $\mathscr{G}_{2}$ is translation-invariant and satisfy that for any $k \in\{1,2\}$ and any $G \in \mathscr{G}_{k}$, there is $j \in\{0,1\} \quad$ satisfying that $G_{2, j}:=\{i \in\{0,1, \ldots\}: 2 j+$ $i \in G\} \in \mathscr{G}_{k}$, and that for any $k \in\{1,2\}$ and any $G \in \mathscr{G}_{k}$,

$$
G_{2}:=\{2 i+j \in\{0,1, \ldots\}: j \in\{0,1\}, i \in G\} \in \mathscr{G}_{k},
$$

then $\Upsilon$ is strong $\left(\mathscr{G}_{1}, \mathscr{G}_{2}\right)$-chaotic if and only if so is $\left.\Upsilon^{2}\right|_{\Lambda_{2}}$.

Proof. We assume that $\Upsilon$ is strong $\left(\mathscr{G}_{1}, \mathscr{G}_{2}\right)$-chaotic.

Claim 3. $\Upsilon^{2}$ is strong $\left(\mathscr{G}_{1}, \mathscr{G}_{2}\right)$-chaotic.

The Proof of Claim 3. Assume that $D \subset H_{1} \times H_{2}$ is a strong $\left(\mathscr{G}_{1}, \mathscr{G}_{2}\right)$-scrambled set of the system $\left(H_{1} \times H_{2}, \Upsilon\right)$. As $\Upsilon$ and $Y^{2}$ are uniformly continuous, for any $t>0$ there is $t^{\prime}>0$ satisfying that $h_{1}, h_{2} \in H_{1} \times H_{2}$ and $\xi\left(h_{1}, h_{2}\right)<t^{\prime}$ imply $\xi\left(\Upsilon\left(h_{1}\right), \Upsilon\left(h_{2}\right)\right)<t$ and $\xi\left(\Upsilon^{2}\left(h_{1}\right), \Upsilon^{2}\left(h_{2}\right)\right)<t$. By hypothesis and the definition, for any $d_{1}, d_{2} \in D$ with $d_{1} \neq d_{2}$, one has that

$$
G=\left\{m \in\{0,1, \ldots\}: \xi\left(\Upsilon^{m}\left(d_{1}\right), \Upsilon^{m}\left(d_{2}\right)\right)<t^{\prime}\right\} \in \mathscr{G}_{1} .
$$

As $\mathscr{G}_{1}$ satisfies that for any $G \in \mathscr{G}_{1}$,

$$
G_{2}:=\{2 i+j \in\{0,1, \ldots\}: j \in\{0,1\}, i \in G\} \in \mathscr{G}_{1},
$$

by the definition there is $j \in\{0,1\}$ satisfying that

$$
G_{2, j}:=\{i \in\{0,1, \ldots\}: 2 j+i \in G\} \in \mathscr{G}_{1} .
$$

By the above argument, one has that

$$
G_{2, j} \subset\left\{m \in\{0,1, \ldots\}: \xi\left(\Upsilon^{2 m+j+2-j}\left(d_{1}\right), \Upsilon^{2 m+j+2-j}\left(d_{2}\right)\right)<t\right\} .
$$

That is,

$$
G_{2, j} \subset\left\{m \in\{0,1, \ldots\}: \xi\left(\Upsilon^{2 m+2}\left(d_{1}\right), \Upsilon^{2 m+2}\left(d_{2}\right)\right)<t\right\} .
$$

So,

$$
\left\{m \in\{0,1, \ldots\}: \xi\left(\Upsilon^{2 m+2}\left(d_{1}\right), \Upsilon^{2 m+2}\left(d_{2}\right)\right)<t\right\} \in \mathscr{G}_{1} .
$$

As $\mathscr{G}_{1}$ is translation-invariant,

$\left\{m \in\{0,1, \ldots\}: \xi\left(\Upsilon^{2 m}\left(d_{1}\right), \Upsilon^{2 m}\left(d_{2}\right)\right)<t\right\} \in \mathscr{G}_{1}$.

By the hypothesis and the definition, there is $\delta>0$ such that for any $d_{1}, d_{2} \in D$ with $d_{1} \neq d_{2}$,

$$
G^{\prime}=\left\{m \in\{0,1, \ldots\}: \xi\left(\Upsilon^{m}\left(d_{1}\right), \Upsilon^{m}\left(d_{2}\right)\right)>\delta\right\} \in \mathscr{G}_{2} .
$$

As $\mathscr{G}_{2}$ satisfies that for any $G \in \mathscr{G}_{2}$, there is $j \in\{0,1\}$ satisfying that $G_{2, j}:=\{i \in\{0,1, \ldots\}: 2 j+i \in G\} \in \mathscr{G}_{2}$, by the definition there is $j \in\{0,1\}$ satisfying that

$$
G_{2, j}^{\prime}:=\left\{i \in\{0,1, \ldots\}: 2 j+i \in G^{\prime}\right\} \in \mathscr{G}_{2} .
$$

As $\Upsilon$ and $\Upsilon^{2}$ are uniformly continuous, for the above $\delta>0$ there is $\delta^{\prime}>0$ satisfying that $h_{1}, h_{2} \in H_{1} \times H_{2}$ and $\xi\left(h_{1}, h_{2}\right) \leq \delta^{\prime}$ imply $\xi\left(\Upsilon\left(h_{1}\right), \Upsilon\left(h_{2}\right)\right) \leq \delta$ and $\xi\left(\Upsilon^{2}\left(h_{1}\right)\right.$, $\left.\Upsilon^{2}\left(h_{2}\right)\right) \leq \delta$. Clearly,

$$
G_{2, j}^{\prime} \subset\left\{m \in\{0,1, \ldots\}: \xi\left(\Upsilon^{2 m}\left(d_{1}\right), \Upsilon^{2 m}\left(d_{2}\right)\right)>\delta^{\prime}\right\},
$$

which means that

$$
\left\{m \in\{0,1, \ldots\}: \xi\left(\Upsilon^{2 m}\left(d_{1}\right), \Upsilon^{2 m}\left(d_{2}\right)\right)>\delta^{\prime}\right\} \in \mathscr{G}_{2} .
$$

Thus, Claim 3 holds.

As $\Upsilon^{2}=\left(f_{1} \circ f_{2}\right) \times\left(f_{2} \circ f_{1}\right)$, by hypothesis, Claim 3, the definition of strong $\left(\mathscr{G}_{1}, \mathscr{G}_{2}\right)$-chaos, and Theorem 2 and its proof, one can easily verify that $f_{1} \circ f_{2}$ and $f_{2} \circ f_{1}$ are strong $\left(\mathscr{G}_{1}, \mathscr{G}_{2}\right)$-chaotic.

Assume that $f_{1} \circ f_{2}$ is strong $\left(\mathscr{G}_{1}, \mathscr{G}_{2}\right)$-chaotic. By the definition, there is an uncountable subset $C \subset H_{1}$ which is strong $\left(\mathscr{G}_{1}, \mathscr{G}_{2}\right)$-scrambled set of $f_{1} \circ f_{2}$. By the proof of Theorem 2, $f_{2}(C)$ is an uncountable and strong $\left(\mathscr{G}_{1}\right.$, $\left.\mathscr{G}_{2}\right)$-scrambled set of $f_{2} \circ f_{1}$. Set $A=\left\{\left(a, g_{2}(a)\right): a \in C\right\}$. Then, $A$ is uncountable. By the above argument, the definition of strong $\left(\mathscr{G}_{1}, \mathscr{G}_{2}\right)$-chaos and the proof of Theorem 2, it is easy to prove that $A$ is a strong $\left(\mathscr{G}_{1}, \mathscr{G}_{2}\right)$-scrambled set of $\left.\Upsilon^{2}\right|_{\Lambda_{2}}$.

Now, we assume that $\left.\Upsilon^{2}\right|_{\Lambda_{2}}$ is strong $\left(\mathscr{G}_{1}, \mathscr{G}_{2}\right)$-chaotic.

Claim 4. $\Upsilon$ is strong $\left(\mathscr{G}_{1}, \mathscr{G}_{2}\right)$-chaotic.

The Proof of Claim 4. By the hypothesis and the definitions, $\Upsilon^{2}$ is strong $\left(\mathscr{G}_{1}, \mathscr{G}_{2}\right)$-chaotic. Assume that $D$ is a strong $\left(\mathscr{G}_{1}, \mathscr{G}_{2}\right)$-scrambled set of the system $\left(H_{1} \times H_{2}, \Upsilon^{2}\right)$. As $\Upsilon^{n}$ is uniformly continuous for any $n \in\{0,1\}$, for any $t>0$ there is $t^{\prime}>0$ satisfying that $h_{1}, h_{2} \in H_{1} \times H_{2}$ and $\xi\left(h_{1}, h_{2}\right)<t^{\prime}$ imply $\xi\left(\Upsilon^{n}\left(h_{1}\right), \Upsilon^{n}\left(h_{2}\right)\right)<t$ for any $n \in\{0,1\}$. By the hypothesis and the definition, for any $d_{1}, d_{2} \in D$ with $d_{1} \neq d_{2}$, one has that 
$G=\left\{m \in\{0,1, \ldots\}: \xi\left(\Upsilon^{2 m}\left(d_{1}\right), \Upsilon^{2 m}\left(d_{2}\right)\right)<t^{\prime}\right\} \in \mathscr{G}_{1}$.

So, for any $m \in G$ and any $n \in\{0,1\}$, we have that

$$
\xi\left(\Upsilon^{2 m+n}\left(d_{1}\right), \Upsilon^{2 m+n}\left(d_{2}\right)\right)<t
$$

As $\mathscr{G}_{1}$ satisfies that for any $G \in \mathscr{G}_{1}$,

$G_{2}:=\{2 i+j \in\{0,1, \ldots\}: j \in\{0,1\}, i \in G\} \in \mathscr{G}_{1}$,

by the definition we have

$$
G_{2}:=\{2 m+n: m \in G, n \in\{0,1\}\} \in \mathscr{G}_{1} .
$$

Clearly,

$$
G_{2} \subset\left\{m \in\{0,1, \ldots\}: \xi\left(\Upsilon^{m}\left(d_{1}\right), \Upsilon^{m}\left(d_{2}\right)\right)<t\right\} .
$$

This means that

$$
\left\{m \in\{0,1, \ldots\}: \xi\left(\Upsilon^{m}\left(d_{1}\right), \Upsilon^{m}\left(d_{2}\right)\right)<t\right\} \in \mathscr{G}_{1} \text {. }
$$

By the hypothesis and the definition, there is $\delta^{\prime}>0$ satisfying that for any $d_{1}, d_{2} \in D$ with $d_{1} \neq d_{2}$,

$$
G^{\prime}=\left\{m \in\{0,1, \ldots\}: \xi\left(\Upsilon^{m}\left(d_{1}\right), \Upsilon^{m}\left(d_{2}\right)\right)>\delta^{\prime}\right\} \in \mathscr{G}_{2} .
$$

As $\Upsilon^{n}$ is uniformly continuous for any $n \in\{0,1\}$, for the above $\delta^{\prime}>0$ there is $\delta>0$ satisfying that $h_{1}, h_{2} \in H_{1} \times H_{2}$ and $\xi\left(\Upsilon^{2}\left(h_{1}\right), \Upsilon^{2}\left(h_{2}\right)\right)>\delta$ imply $\xi\left(\Upsilon^{n}\left(h_{1}\right), \Upsilon^{n}\left(h_{2}\right)\right)>\delta$ for any $n \in\{0,1\}$. So, for any $m \in G^{\prime}$ and any $n \in\{0,1\}$, we have that

$$
\xi\left(\Upsilon^{2(m-1)+n}\left(d_{1}\right), \Upsilon^{2(m-1)+n}\left(d_{2}\right)\right)>\delta .
$$

As $\mathscr{G}_{2}$ is translation-invariant, $G^{\prime}-1 \in \mathscr{G}_{2}$. As $\mathscr{G}_{2}$ satisfies that for any $G \in \mathscr{G}_{2}$,

$$
\begin{array}{r}
G_{2}:=\{2 i+j \in\{0,1, \ldots\}: j \in\{0,1\}, i \in G\} \in \mathscr{G}_{2}, \\
\left(G^{\prime}-1\right)_{2}:=\left\{2(m-1)+n: m-1 \in G^{\prime}-1, n \in\{0,1\}\right\} \in \mathscr{G}_{2} .
\end{array}
$$

Clearly,

$$
\left(G^{\prime}-1\right)_{2} \subset\left\{m \in\{0,1, \ldots\}: \xi\left(\Upsilon^{m}\left(d_{1}\right), \Upsilon^{m}\left(d_{2}\right)\right)>\delta\right\}
$$

which means that

$$
\left\{m \in\{0,1, \ldots\}: \xi\left(\Upsilon^{m}\left(d_{1}\right), \Upsilon^{m}\left(d_{2}\right)\right)>\delta\right\} \in \mathscr{G}_{2} .
$$

Thus, Claim 4 holds.

Consequently, Theorem 4 is true.

Corollary 2. Let $\Upsilon(a, b)=\left(f_{1}(b), f_{2}(a)\right)$ be a Cournot map on the product space $H_{1} \times H_{2}$. Then, for any $a, b \in[0,1]$, $\Upsilon$ is $(\bar{M}(a), \bar{M}(b))$-chaotic (resp. strong $(\bar{M}(a)$, $\bar{M}(b))$-chaotic) if and only if so is $\left.Y^{2}\right|_{\Lambda_{2}}$.

Proof. We have the following two claims.
Claim 5. For any $t \in[0,1], \bar{M}(t)$ satisfies that for any $G \in \mathscr{G}$, there is $j \in\{0,1\}$ such that $G_{2, j}:=\{i \in\{0,1$, $\ldots\}: 2 j+i \in G\} \in \mathscr{G}$.

The Proof of Claim 5. It is clear that $\bar{M}(0)=\mathscr{B}$ and that if $G \in \mathscr{B}$, then there is $j \in\{0,1\}$ satisfying that $G_{2, j} \in \mathscr{B}$. Assume that there is $t \in(0,1]$ such that $\bar{M}(t)$ does not have the property $P(2)$. By this assumption and the definition, there is $G \in \bar{M}(t)$ such that for any $j \in\{0,1\}, \bar{\mu}\left(G_{2, j}\right)$ $=e_{j}<t$. Choose $\delta_{j} \in\left(0, t-e_{j}\right)$ for any $j \in\{0,1\}$. As $\bar{\mu}\left(G_{2, j}\right)=e_{j}<t$ for any $j \in\{0,1\}$, by the definition there is an integer $M>0$ such that for any $j \in\{0,1\}$ and any integer $m \geq M, \sharp\left(G_{2, j} \cap\{1,2, \ldots, m\}\right)<\left(t-\delta_{j}\right) m$. This implies that

$$
\sharp\left(\left(\{1,2, \ldots\} \backslash G_{2, j}\right) \cap\{1,2, \ldots, m\}\right)>m-\left(t-\delta_{j}\right) m \text {. }
$$

Let $n \in\{2 M+1,2 M+1, \cdots\}$ and write $n=2[n / 2]+l_{n}$, where $[n / 2]$ is the integral part of $n / 2$ and $l_{n} \in\{0,1\}$. By the definition, $i \notin G_{2, j}$ implies $2 i+j \notin G$ for any $j \in\{0,1\}$. Obviously, if $j_{1}, j_{2} \in\{0,1\}$ and $j_{1} \neq j_{2}$ then $2 i_{1}+j_{1} \neq 2 i_{2}+j_{2}$ for any $i_{1}, i_{2} \in\{0,1, \ldots, m\}$. So,

$$
\begin{aligned}
& \sharp((\{1,2, \ldots\} \backslash G) \cap\{1,2, \ldots, n\}) \geq \sharp\left(A_{0}\right)+\sharp\left(A_{1}\right)>\left[\frac{n}{2}\right] \\
& -\left(t-e_{0}\right)\left[\frac{n}{2}\right]+\left[\frac{n}{2}\right]-\left(t-e_{1}\right)\left[\frac{n}{2}\right],
\end{aligned}
$$

where

$$
\begin{aligned}
& A_{0}=\left(\{1,2, \ldots\} \backslash G_{2,0}\right) \cap\left\{1,2, \ldots,\left[\frac{n}{2}\right]\right\}, \\
& A_{1}=\left(\{1,2, \ldots\} \backslash G_{2,1}\right) \cap\left\{1,2, \ldots,\left[\frac{n}{2}\right]\right\} .
\end{aligned}
$$

This implies that

$\sharp(G \cap\{1,2, \ldots, n\})<n-\left(\left[\frac{n}{2}\right]-\left(t-e_{0}\right)\left[\frac{n}{2}\right]+\left[\frac{n}{2}\right]-\left(t-e_{1}\right)\left[\frac{n}{2}\right]\right)$.

Set $\delta=\min \left\{\delta_{0}, \delta_{1}\right\}$. As

$$
\begin{aligned}
\bar{\mu}(G)= & \limsup _{n \longrightarrow \infty} \frac{1}{n} \sharp(G \cap\{1,2, \ldots, n\}) \leq \limsup _{n \longrightarrow \infty} \frac{1}{n} \\
& \cdot\left(n-\left(\left[\frac{n}{2}\right]-\left(t-e_{0}\right)\left[\frac{n}{2}\right]+\left[\frac{n}{2}\right]-\left(t-e_{1}\right)\left[\frac{n}{2}\right]\right)\right), \\
\bar{\mu}(G) \leq & \frac{1}{n}\left(n-2\left(\left[\frac{n}{2}\right]-(t-\delta)\left[\frac{n}{2}\right]\right)\right) .
\end{aligned}
$$

By $n=2[n / 2]+l_{n}$,

$$
\bar{\mu}(G) \leq t-\delta .
$$

This is a contraction. Consequently, Claim 5 holds.

Claim 6. For any $t \in[0,1], \bar{M}(t)$ satisfies that for any $G \in \mathscr{G}$ : 


$$
G_{2}:=\{2 i+j \in\{0,1, \ldots\}: j \in\{0,1\}, i \in G\} \in \mathscr{G} .
$$

The Proof of Claim 6. It is obvious that $\bar{M}(0)=\mathscr{B}$, and that if $G \in \mathscr{B}$, then $G_{2} \in \mathscr{B}$. Assume that there is $t \in(0,1]$ such that $\bar{M}(t)$ does not have the property $Q(2)$. By this assumption and the definition, there is $G \in \bar{M}(t)$ such that $\bar{\mu}\left(G_{2}\right)=e<t$. Choose $\delta \in(0, t-e)$. As $\bar{\mu}\left(G_{2}\right)=e<t$, by the definition there is an integer $M>0$ such that for any integer $m \geq M, \sharp\left(G_{2} \cap\{1,2, \ldots, m\}\right)<(t-\delta) m$. Let $n \in\{2 M+1,2$ $M+1, \cdots\}$ and write $n=2[n / 2]+l_{n}$, where $[n / 2]$ is the integral part of $n / 2$ and $l_{n} \in\{0,1\}$. By the definition, $i \in G$ implies $2 i+j \in G_{2}$ for any $j \in\{0,1\}$. Obviously, if $i_{1}, i_{2} \in G$ and $j_{1} \neq j_{2}$, then $2 i_{1}+j_{1} \neq 2 i_{2}+j_{2}$ for any $j_{1}, j_{2} \in\{0,1\}$. So,

$$
2 \sharp\left(G \cap\left\{1,2, \ldots,\left[\frac{n}{2}\right]\right\}\right) \leq \sharp\left(G_{2} \cap\{1,2, \ldots, n\}\right)<n(t-\delta) .
$$

This implies that

$$
\sharp\left(G \cap\left\{1,2, \ldots,\left[\frac{n}{2}\right]\right\}\right)<\frac{1}{2}\left(2\left[\frac{n}{2}\right]+l_{n}\right)(t-\delta) .
$$

Consequently,

$$
\bar{\mu}(G) \leq \lim _{n \longrightarrow \infty} \frac{1}{2[n / 2]}\left(2\left[\frac{n}{2}\right]+l_{n}\right)(t-\delta)=t-\delta<t .
$$

This is a contraction. Consequently, Claim 5 is true.

From the above two claims we know that $\bar{M}(t)$ satisfies the conditions of Theorems 3 and 4 for any $t \in[0,1]$. Thus, by these two theorems one can see that Corollary 2 holds.

Theorem 5. Let $\Upsilon(a, b)=\left(f_{1}(b), f_{2}(a)\right)$ be a Cournot map on the product space $H_{1} \times H_{2}$. If $\mathscr{G}_{1}$ and $\mathscr{G}_{2}$ are two Furstenberg families such that $\mathscr{G}_{2}$ is translation-invariant, then $\Upsilon$ is $\left(\mathscr{G}_{1}, \mathscr{G}_{2}\right)$-chaotic (resp. strong $\left(\mathscr{G}_{1}, \mathscr{G}_{2}\right)$-chaotic) if and only if so is $\left.Y^{2}\right|_{\Lambda_{1}}$.

Proof. The proof is similar to those of Theorems 3 and 4 and is omitted.

Corollary 3. Let $\Upsilon(a, b)=\left(f_{1}(b), f_{2}(a)\right)$ be a Cournot map on the product space $H_{1} \times H_{2}$. Then, for any $a, b \in[0,1]$, $\Upsilon$ is $(\bar{M}(a), \bar{M}(b))$-chaotic (resp. strong $(\bar{M}(a), \bar{M}(b))$ -chaotic) if and only if so is $\left.Y^{2}\right|_{\Lambda_{1}}$.

Proof. By Theorem 5 and the proof of Corollary 2 one can easily see that Corollary 3 holds.

Theorem 6. Let $\Upsilon(a, b)=\left(f_{1}(b), f_{2}(a)\right)$ be a Cournot map on the product space $H_{1} \times H_{2}$. If $\mathscr{G}_{1}$ and $\mathscr{G}_{2}$ are two Furstenberg families such that $\mathscr{G}_{2}$ is translation-invariant, then $\Upsilon$ is $\left(\mathscr{G}_{1}, \mathscr{G}_{2}\right)$-chaotic (resp. strong $\left(\mathscr{G}_{1}, \mathscr{G}_{2}\right)$-chaotic) if and only if so is $\left.Y^{2}\right|_{\Lambda_{1} \cup \Lambda_{2}}$.

Proof. By hypothesis, the definitions of $\Lambda_{1}, \Lambda_{2}$, and $\left(\mathscr{G}_{1}, \mathscr{G}_{2}\right)$-chaos (resp. strong $\left(\mathscr{G}_{1}, \mathscr{G}_{2}\right)$-chaos) and Theorems 3 and 4 , it is easily seen that $\Upsilon$ is $\left(\mathscr{G}_{1}, \mathscr{G}_{2}\right)$-chaotic (resp. strong $\left(\mathscr{G}_{1}, \mathscr{G}_{2}\right)$-chaotic) if and only if so is $\left.\Upsilon^{2}\right|_{\Lambda_{1} \cup \Lambda_{2}}$.
Corollary 4. Let $\Upsilon(a, b)=\left(f_{1}(b), f_{2}(a)\right)$ be a Cournot map on the product space $H_{1} \times H_{2}$. Then, for any $a, b \in[0,1]$, $\Upsilon$ is $(\bar{M}(a), \bar{M}(b))$-chaotic (resp. strong $(\bar{M}(a), \bar{M}(b))$ -chaotic) if and only if so is $\left.Y^{2}\right|_{\Lambda_{1} \cup \Lambda_{2}}$.

Proof. By Theorem 6 and the proof of Corollary 2 one can easily see that Corollary 4 holds.

\section{Data Availability}

The data used to support the findings of this study are available from the corresponding author upon request.

\section{Conflicts of Interest}

The authors declare that they have no conflicts of interest.

\section{Authors' Contributions}

All authors contributed equally to this work. All authors read and approved the final manuscript.

\section{Acknowledgments}

This project was supported by the Opening Project of Artificial Intelligence Key Laboratory of Sichuan Province (No. 2018RZJ03) and the Opening Project of Bridge Nondestruction Detecting and Engineering Computing Key Laboratory of Sichuan Province (2018QZJ03).

\section{References}

[1] T. Puu and I. Sushko, Oligopoly Dynamics: Models and Tools, Springer, New York, NY, USA, 2002.

[2] T.-Y. Li and J. A. Yorke, "Period three implies chaos," The American Mathematical Monthly, vol. 82, no. 10, pp. 985-992, 1975.

[3] E. Akin and S. Kolyada, "Li-Yorke sensitivity," Nonlinearity, vol. 16, no. 4, pp. 1421-1433, 2003.

[4] B. Schweizer and J. Smital, "Measures of chaos and a spectral decomposition of dynamical systems on the interval," Transactions of the American Mathematical Society, vol. 344, no. 2, pp. 737-754, 1994.

[5] P. Oprocha and P. Wilczyński, "Shift spaces and distributional chaos," Chaos, Solitons \& Fractals, vol. 31, no. 2, pp. 347-355, 2007.

[6] J. Smítal and M. Štefánková, "Distributional chaos for triangular maps," Chaos, Solitons \& Fractals, vol. 21, no. 5, pp. 1125-1128, 2004.

[7] R. Pikula, "On some notions of chaos in dimension zero," Colloquium Mathematicum, vol. 107, pp. 167-177, 2007.

[8] L. Wang, G. Huang, and S. Huan, "Distributional chaos in a sequence," Nonlinear Analysis: Theory, Methods \& Applications, vol. 67, no. 7, pp. 2131-2136, 2007.

[9] J. S. Cánovas and M. Rúiz Marín, "Chaos on MPE-sets of duopoly games," Chaos, Solitons \& Fractals, vol. 19, no. 1, pp. 179-183, 2004.

[10] J. S. Cánovas, M. Ruíz Marín, and G. Soler, "Ditributional chaos of duopoly games," Advanced Nonlinear Studies, vol. 1, pp. 79-87, 2001. 
[11] J. S. Cánovas, "Chaos in duopoly games," Nonlinear Studies, vol. 7, pp. 97-104, 2000.

[12] R.-A. Dana and L. Montrucchio, "Dynamic complexity in duopoly games," Journal of Economic Theory, vol. 40, no. 1, pp. 40-56, 1986.

[13] T. Lu and P. Zhu, "Further discussion on chaos in duopoly games," Chaos, Solitons \& Fractals, vol. 52, pp. 45-48, 2013.

[14] G. I. Bischi, C. Mammana, and L. Gardini, "Multistability and cyclic attractors in duopoly games," Chaos, Solitons and Fractals, vol. 11, pp. 543-564, 2000.

[15] M. Kopel, "Simple and complex adjustment dynamics in Cournot duopoly models," Chaos, Solitons \& Fractals, vol. 7, no. 12 , pp. 2031-2048, 1996.

[16] T. Puu, "Chaos in duopoly pricing," Chaos, Solitons \& Fractals, vol. 1, no. 6, pp. 573-581, 1991.

[17] D. Rand, "Exotic phenomena in games and duopoly models," Journal of Mathematical Economics, vol. 5, no. 2, pp. 173-184, 1978.

[18] E. Akin, Recurrence in Topological Dynamics: Furstenberg and Ellis Action, Plenum Press, New York, NY, USA, 1997.

[19] F. Tan and J. Xiong, "Chaos via Furstenberg family couple," Topology and Its Applications, vol. 156, no. 3, pp. 525-532, 2009. 


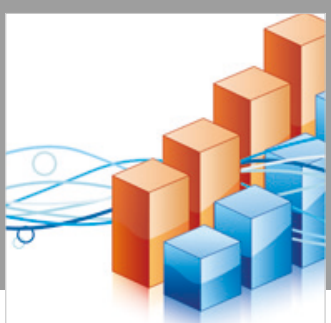

Advances in

Operations Research

\section{-n-m}
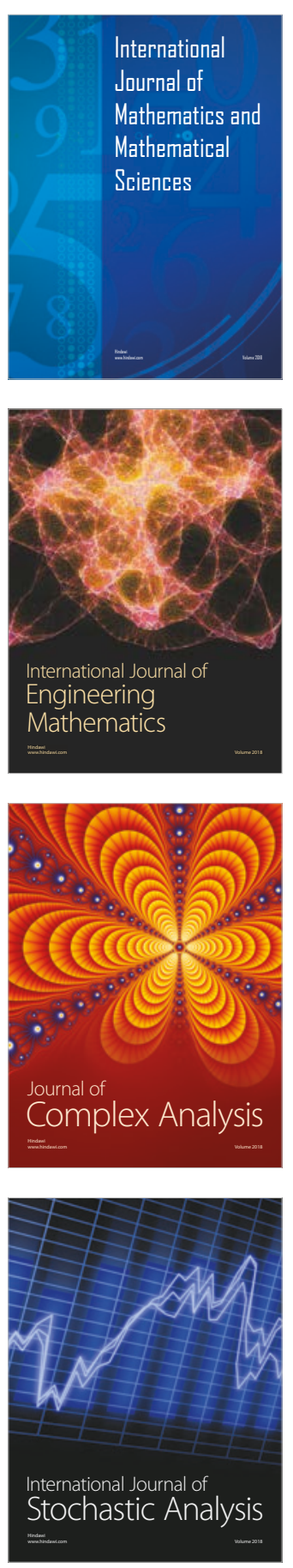
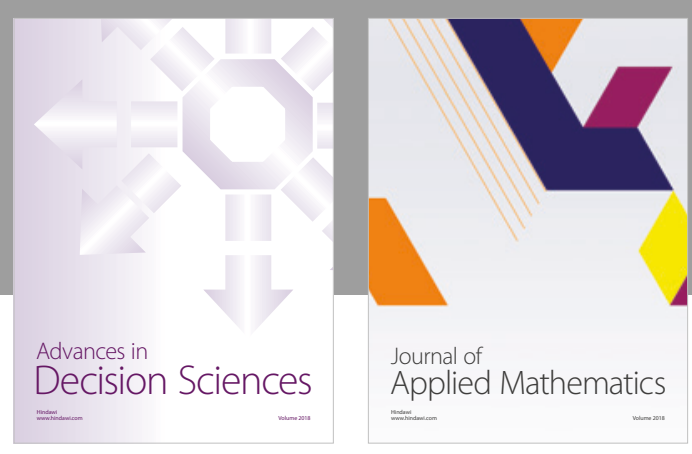

Journal of

Applied Mathematics
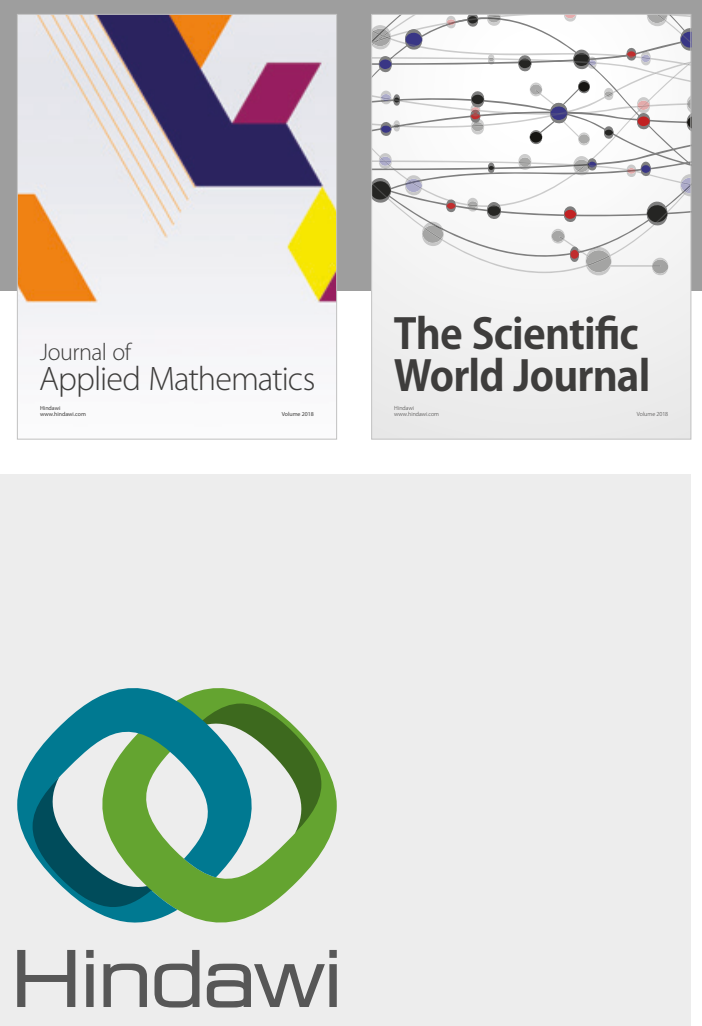

Submit your manuscripts at

www.hindawi.com

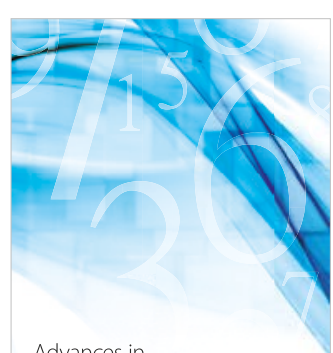

Advances in
Numerical Analysis
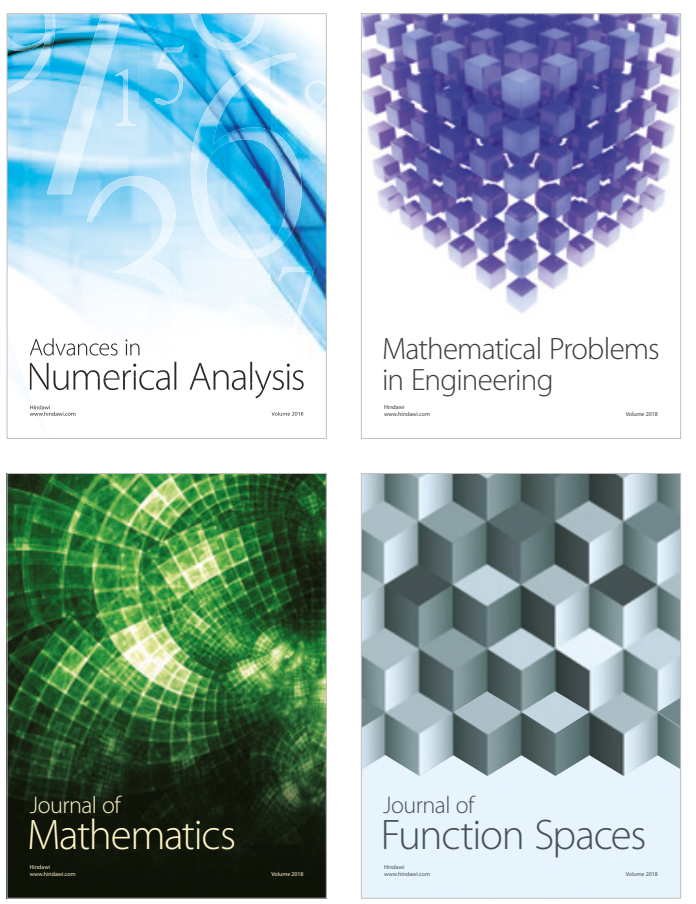

Mathematical Problems in Engineering

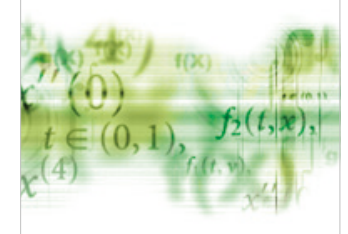

International Journal of

Differential Equations

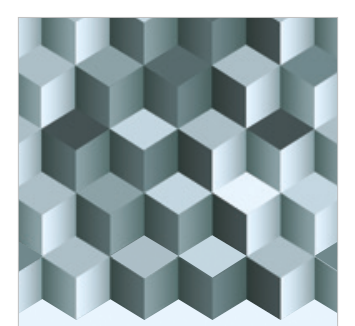

Journal of

Function Spaces
The Scientific

World Journal

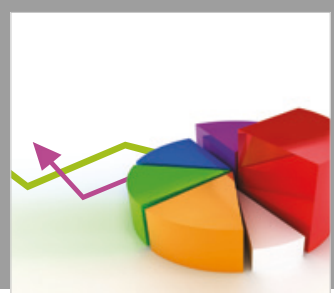

Journal of

Probability and Statistics
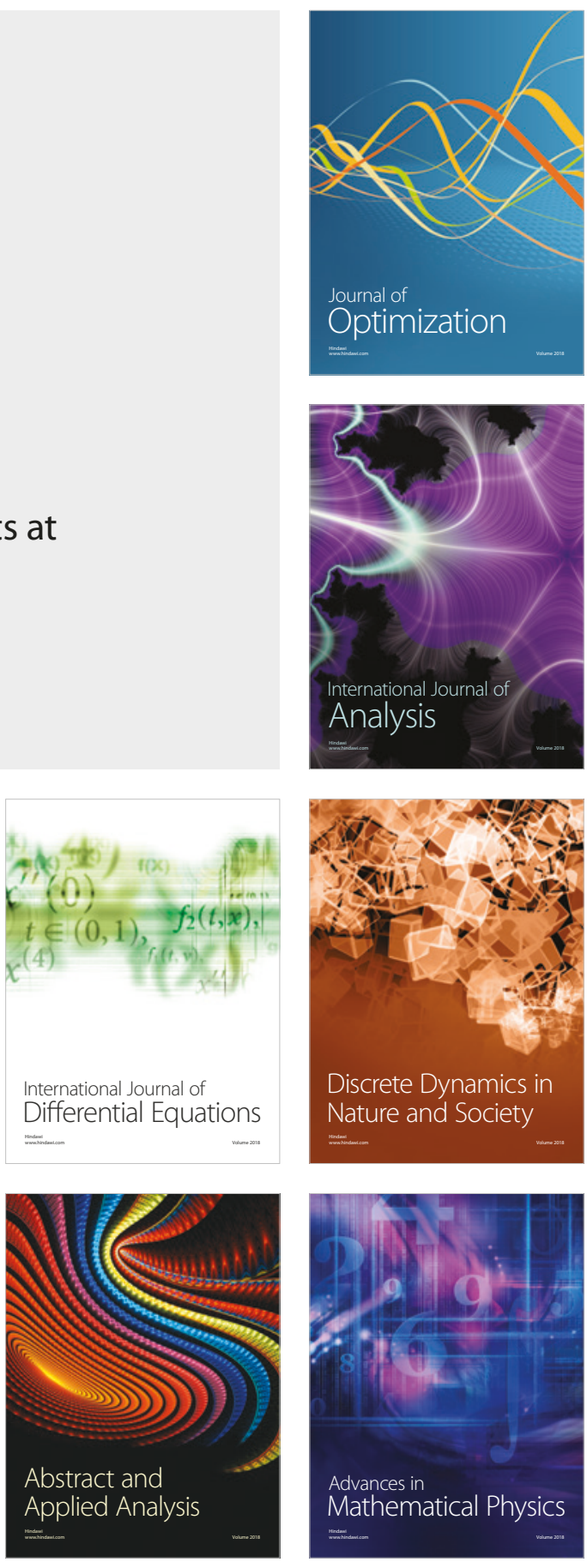